Pacific Journal of Mathematics

THE EXISTENCE OF STRONG LIFTINGS FOR TOTALLY 


\title{
THE EXISTENCE OF STRONG LIFTINGS FOR TOTALLY ORDERED MEASURE SPACES
}

\author{
A. Sapounakis
}

Let $X$ be a totally ordered space, $\mu$ a finite Borel measure on $X$ with full support, and $\mathcal{F}$ the $\sigma$-algebra of all $\mu^{*}$-measurable subsets of $X$. Then there exists a lifting $\rho: \mathscr{F} \rightarrow \mathscr{F}$ which satisfies $U \subset \rho(U)$ for every open subset $U$ of $X$.

Assume that $X$ is a topological space and $\mu$ a finite, Borel measure on $X$ with full support. We are interested in finding conditions for the topology of $X$, which insure the existence of strong liftings for the associated topological measure space. In [8] Losert has given an example showing that this is not always possible even if $X$ is compact. On the other hand Graf has proved that strong liftings always exist for measures on second countable spaces [6]. Other positive results on the existence of strong liftings are given in [2] and [4].

In this paper we show that every totally ordered measure space admits a strong lifting. Moreover we prove that if $\mu$ is a Radon, non-atomic measure on a totally ordered space then every lifting of the associated measure space is almost strong, if and only if, the set of all two sided limit points of the support of $\mu$ is $\mu^{*}$-measurable with full measure.

1. Preliminaries and notation. Throughout $X$ will be a set and " $\leq$ " a total order on $X$. If $x, y$ are two points of $X$, let $x<y$ means that $x \leq y$ and $x \neq y$. Let $(-\infty, x)=\{z \in X: z<x\},(x,+\infty)=\{z \in X$ : $x<z\}$ and $(x, y)=(-\infty, y) \cap(x,+\infty)$. Assume that $Y \subset X$ and $y \in Y$. We say that $y$ is a left limit point of $Y$ if $(-\infty, y) \cap Y \neq \varnothing$ and there is no $z$ in $Y$ such that $z<y$ and $(z, y) \cap Y=\varnothing$. Analogous is the definition of the right limit point. A point $y$ in $Y$ is said to be a two sided limit point of $Y$ if it is both a left and right limit point of $Y$.

We say that $(X, \leq)$ is a totally ordered (topological) space if its topology is generated by all the intervals of the form $(-\infty, x),(x,+\infty)$. By a measure on $X$ we mean a finite, non-negative, countably additive set function defined on the Borel sets of $X$. A measure $\mu$ on $X$ is said to be Radon if it is inner approximated by the compact sets of $X$. The support $S_{\mu}$ of a measure $\mu$ on $X$ is defined by

$$
S_{\mu}=\cap\{F: F \text { closed subset of } X \text { such that } \mu(F)=\mu(X)\} \text {. }
$$


Clearly $S_{\mu}$ is a closed subset of $X$ and if $\mu(X)=\mu\left(S_{\mu}\right), S_{\mu}$ satisfies the countable chain condition.

A totally ordered measure space is the quadriple $(X, \leq, \mathscr{F}, \mu)$, where $(X, \leq)$ is a totally ordered space, $\mu$ is a measure on $X$ and $\mathscr{F}$ is the completion of the Borel $\sigma$-algebra of $X$ w.r.t. $\mu$.

A map $\rho: \mathcal{F} \rightarrow \mathscr{F}$ is called lifting [7] if for all $A, B \in \mathscr{F}$

(i) $\mu(A \Delta \rho(A))=0$,

(ii) $\mu(A \Delta B)=0 \Rightarrow \rho(A)=\rho(B)$,

(iii) $\rho(\varnothing)=\varnothing, \rho(X)=X$,

(iv) $\rho(A \cup B)=\rho(A) \cup \rho(B)$,

(v) $\rho(A \cap B)=\rho(A) \cap \rho(B)$.

If $\rho$ satisfies only the properties (i), (ii), (iii), (iv) (resp. (i), (ii), (iii), (vi)) is called an upper (resp. lower) density.

A lifting $\rho$ is said to be

(a) strong, if $U \subset \rho(U)$ for all open subsets $U$ of $X$,

(b) almost strong if there is a set $N \in \mathscr{F}$ with $\mu(N)=0$, and $U \subset N$ $\cup \rho(U)$ for all open subsets $U$ of $X$.

2. The results. We start with the main result of this paper, the proof of which is divided in three steps. The proof of the first step is based on an idea of [[7], Example 3, page 122], and on the arguments used in the proof of Theorem 3.2 in [10].

THEOREM 2.1. Let $(X, \leq, \mathscr{F}, \mu)$ be a totally ordered measure space such that $X=S_{\mu}$. Then there exists a strong lifting $\rho: \mathcal{F} \rightarrow \mathscr{F}$.

Proof. Without loss of generality we may assume that $\mu(X)=1$.

Step I. We prove the theorem in the case when $S$ is compact and $\mu$ a Radon, non-atomic measure.

Let $f$ be the distribution function of $\mu$ defined by $f(x)=\mu((-\infty, x))$ for every $x \in X$. Then $f$ is an ordered preserving, continuous function from $X$ onto the unit interval $[0,1]$, such that $f^{-1} f(B)-B$ is at most countable for every Borel subset $B$ of $X$ (see proof of Theorem 3.2 in [10]). Moreover if $\lambda$ denotes the Lebesgue measure on $[0,1]$ we can easily verify that $\lambda(B)=\mu\left(f^{-1}(B)\right)$ for every Borel subset $B$ of $[0,1]$.

We show that for every $F \in \mathscr{F}$

$$
f(F) \text { is Lebesgue measurable and } \lambda(f(F))=\mu(F) .
$$

Indeed since $X$ is hereditary Lindelöf [[3], Theorem 2.2], every Borel subset $F$ of $X$ is $\kappa$-analytic and so $f(F)$ is an absolutely measurable subset of $[0,1]$. Thus $f(F)$ is Lebesgue measurable and since $f^{-1} f(F)-F$ is at most countable we deduce that $\lambda(f(F))=\mu(F)$. This shows that $(*)$ is true for Borel subsets of $X$ and can easily be extended for every $F$ in $\mathscr{F}$. 
Now let $L_{1}$ (resp. $L_{2}$ ) be the set of all left (resp. right) limit points of $X$. Clearly since $X$ has no isolated points we have that $X=L_{1} \cup L_{2}$. We consider each $L_{i}(i=1,2)$ with the order topology, given by the restriction of the ordering on $L_{i}$. Let $f_{i}$ be the restriction of $f$ on $L_{i}$. Then since $f_{1}$ (resp. $f_{2}$ ) is an ordered preserving, one-to-one map from $L_{1}$ (resp. $L_{2}$ ) on $(0,1]$ (resp. $[0,1)$ ), we may easily conclude that $f_{1}$ (resp. $f_{2}$ ) is an homeomorphism.

Let $\lambda_{i}$, be the measure on $L_{i}(i=1,2)$ defined by $\lambda_{i}(E)=\lambda\left(f_{i}(E)\right)$ for every Borel $E \subset L_{i}$, and $\mathscr{F}_{i}$ the completion of the Borel $\sigma$-algebra of $L_{i}$ w.r.t. $\lambda_{i}$. Then using $(*)$ we can easily deduce that

(1) If $F \in \mathscr{F}$ then $F \cap L_{i} \in \mathscr{F}_{i}$ for $i=1,2$.

(2) For every $A \subset X, \mu^{*}(A)=0$ if and only if $\lambda_{i}^{*}\left(A \cap L_{i}\right)=0$ for every $i=1,2$.

(3) $\lambda_{1}^{*}(A)=\lambda_{2}^{*}(A)$ for every $A \subset L_{1} \cap L_{2}$.

Now by [[7], Theorem 6, page 123] there are strong liftings $\rho_{1}, \rho_{2}$ of $\mathscr{F}_{1}, \mathscr{F}_{2}$ respectively, such that

$$
A \subset \rho_{1}(A) \text { for every left-open right-closed interval } A \text { of } L_{1}
$$

and

$B \subset \rho_{2}(B)$ for every left-closed right-open interval $B$ of $L_{2}$.

We define $\theta$ on $\mathcal{F}$ by

$$
\theta(F)=\rho_{1}\left(F \cap L_{1}\right) \cup \rho_{1}\left(F \cap L_{2}\right) .
$$

Then using (1), (2) and (3) we may easily check that $\theta$ is an upper density of $\mathscr{F}$. Let $\theta^{\prime}$ be the associated to $\theta$ lower density of $\mathscr{F}$ defined by $\theta^{\prime}(F)=X-\theta(X-F)$. Then

$$
\theta^{\prime}(F)=\left(\rho_{1}\left(F \cap L_{1}\right) \cup X-L_{1}\right) \cap\left(\rho_{2}\left(F \cap L_{2}\right) \cup X-L_{2}\right) .
$$

We show that $U \subset \theta^{\prime}(U)$, where $U$ is either of the form $(-\infty, x)$ or $(x,+\infty)$. Indeed if $U=(-\infty, x)$, then $U \cap L_{1}$ is either an open interval of $L_{1}$ (if $x \in L_{1}$ ) or a left-open right-closed interval of $L_{1}$ (if $x \in L_{2}$ ). It follows that $U \cap L_{1} \subset \rho_{1}\left(U \cap L_{1}\right)$. On the other hand $U \cap L_{2}$ is always a left-closed right-open interval of $L_{2}$ and so $U \cap L_{2} \subset \rho_{2}\left(U \cap L_{2}\right)$. Thus $U \subset \theta^{\prime}(U)$. In the same way we show that $(x,+\infty) \subset \theta^{\prime}((x,+\infty))$. By [[7], corollary page 58] there exists a lifting $\rho$ of $\mathscr{F}$ such that $\theta^{\prime}(F) \subset \rho(F)$ $\subset \theta(F)$ for every $F \in \mathscr{F}$. It follows that $\rho$ is a strong lifting of $\mathscr{F}$.

Step II. Here we assume that $X$ is compact and $\mu$ Radon.

Set $Y=\{x \in X: \mu(\{x\})>0\}$. Clearly $Y$ is at most countable. Let $\nu$ be the Radon, non-atomic measure on $X$ defined by $\nu(B)=$ $\mu(B \cap X-Y)$ for every Borel $B \subset X$. Since the support $S_{\nu}$ of $\nu$ is a compact totally order space, under the subspace topology, by step I there 
exists a strong lifting for the restriction of $\nu$ on $S_{\nu}$. Let $Z=S_{\nu} \cap(X-Y)$. Clearly $\nu(Z)=\nu\left(S_{\nu}\right)$ and since $\mu, \nu$ coincide on the Borel subsets of $Z$, there exists a strong lifting $\rho^{\prime}$ for the restriction of $\mu$ on $Z$.

Let $T=Z \cup Y$. We define $\rho$ on $\mathscr{F}_{T}$, the class of all $\mu^{*}$-measurable subsets of $T$, by

$$
\rho(E)=\rho^{\prime}(E \cap Z) \cup(E \cap Y) .
$$

It follows that $\rho$ is a strong lifting for the restriction of $\mu$ on $T$ and since $\mu(T)=\mu(X)$ using a standard argument ([7], Remark 1, page 127) we can find a strong lifting of $\mathscr{F}$.

Step III. The general case.

Let $X^{*}$ be the totally ordered compactification of $X$ (see [5], §6). We define a measure $\bar{\mu}$ on $X^{*}$ by $\bar{\mu}(B)=\mu(B \cap X)$, for every Borel $B \subset X^{*}$. We note that $\bar{\mu}$ has full support in $X^{*}$ and thus $X^{*}$ is hereditary Lindelöf [3]. It follows easily that $\bar{\mu}$ is a Radon measure on $X^{*}$.

Let $\overline{\mathcal{F}}$ be the $\sigma$-algebra of all $\bar{\mu}^{*}$-measurable subsets of $X^{*}$. Then we have $F \in \mathscr{F}$ if and only if there is $E \in \overline{\mathscr{F}}$ such that $F=E \cap X$. By Step II, there exists a strong lifting $\bar{\rho}$ of $\overline{\mathscr{F}}$. We define $\rho$ on $\mathscr{F}$ by

$$
\rho(F)=\bar{\rho}(E) \cap X
$$

where $E \in \overline{\mathscr{F}}$ such that $F=E \cap X$. It follows that $\rho$ is a strong lifting of $\mathscr{F}$ and hence the theorem is completely proved.

As measures on totally ordered spaces share a number of properties with measures on metric spaces [10], it is natural to ask whether every lifting of $(X, \leq, \mathscr{F}, \mu)$ is almost strong. The answer is given by using the next theorem, the proof of which uses an argument of [[1], Theorem 3.1] and some already familiar techniques from Theorem 2.1.

THEOREM 2.2. Let $(X, \leq, \mathscr{F}, \mu)$ be a totally ordered measure space, where $\mu$ is a Radon, non-atomic measure on $X$. Then the following are equivalent

(i) Every lifting of $\mathcal{F}$ is almost strong.

(ii) The set $L$ of all two sided limit points of $S_{\mu}$ is $\mu^{*}$-measurable with full measure.

Proof. We first prove the theorem in the case when $X$ is compact. Also in this case without loss of generality we may assume that $\mu$ is a probability measure with full support.

(i) $\Rightarrow$ (ii) As in the Step I of Theorem 2.1 we denote by $f$ the distribution function of $\mu$. We define $\rho$ on $\mathscr{F}$ by

$$
\rho(E)=f^{-1}\left(\rho^{\prime}(f(E))\right)
$$


where $\rho^{\prime}$ is a lifting of the Lebesgue measure on $[0,1]$. Clearly $\rho$ is a lifting of $\mathscr{F}$ and so there exists a $G_{\delta}$ subset $N$ of $X$ such that $\mu(N)=0$ and $U \subset \rho(U) \cup N$ for every open subset $U$ of $X$.

We will prove that $X-N$ contains at most countable many non-two sided limit points of $X$. Assume that this is not true, without loss of generality let $\left\{x_{i}\right\}_{i \in I}$ be an incountable set of non-left limit points of $X-N$. Let $y_{i} \in X$ such that $y_{i}<x_{i}$ and $\left(y_{i}, x_{i}\right)=\varnothing$. Then $y_{i} \in N$ for each $i$, because otherwise $y_{i} \in \rho\left(\left(-\infty, x_{i}\right)\right)$ and if $t_{i}=f\left(x_{i}\right)=f\left(y_{i}\right)$ we have that

$$
t_{i} \in \rho^{\prime}\left(f\left(-\infty, x_{i}\right)\right) \cap \rho^{\prime}\left(f\left(y_{i},+\infty\right)\right),
$$

while

$$
\rho^{\prime}\left(f\left(-\infty, x_{i}\right)\right) \cap \rho^{\prime}\left(f\left(y_{i},+\infty\right)\right)=\rho^{\prime}\left(\left\{t_{i}\right\}\right)=\varnothing .
$$

Now since $N$ is a $G_{\delta}$ subset of $X$ we may find an open subset $U$ of $X$ such that $N \subset U$ and $x_{i} \notin U$ for uncountable many $i$. Further since $X$ is hereditary Lindelöf there is a sequence $\left\{\left(a_{n}, b_{n}\right)\right\}$ of open intervals in $X$ such that $U=\cup_{n=1}^{\infty}\left(a_{n}, b_{n}\right)$. Then if we pick an $i$ such that $x_{i} \notin U$ and $x_{i} \neq b_{n}$ for all $n$, we get that $y_{i} \notin U$, which is a contradiction. This shows that $L \in \mathscr{F}$ and $\mu(L)=\mu(X)$.

(ii) $\Rightarrow$ (i) Let $\rho$ be a lifting of $\mathscr{F}$. We define an upper density $\theta$ of the Lebesgue measure space by

$$
\theta(E)=f\left(\rho\left(f^{-1}(E)\right)\right)
$$

where $f$ again denotes the distribution function of $\mu$. Clearly since every lifting of the Lebesgue measure space is almost strong we can find a set $N \subset[0,1]$ such that $\lambda(N)=0$ and $V \subset \theta(V) \cup N$ for every open $V \subset$ $[0,1]$. It follows easily that

$$
U \subset \rho(U) \cup f^{-1}(N) \cup(X-L)
$$

for every open subset $U$ of $X$. This shows that $\rho$ is almost strong.

We now consider the general case. Let $X^{*}$ be the totally ordered compactification of $X$ and $\bar{\mu}$ the induced by $\mu$ Radon measure on $X^{*}$. Clearly $\bar{\mu}$ is non-atomic and $S_{\mu}=S_{\bar{\mu}} \cap X$.

Let $\bar{L}$ be the set of all two sided limit points of $S_{\bar{\mu}}$ and $\bar{F}$ the completion of the Borel $\sigma$-algebra of $X^{*}$ w.r.t. $\bar{\mu}$. Clearly since $\mu$ is Radon, $X$ is $\bar{\mu}^{*}$-measurable with full $\bar{\mu}$-measure and so every lifting of $\overline{\mathscr{F}}$ is almost strong, if and only if, every lifting of $\mathscr{F}$ is almost strong. It remains to prove that $L$ is $\mu^{*}$-measurable with full measure, if and only if, $\bar{L}$ is $\bar{\mu}^{*}$-measurable with full measure. Indeed assume that $L \in \mathcal{F}$ and $\mu(L)=$ $\mu(X)$. Then using similar arguments as before we can show that $K \cap\left(X^{*}\right.$ $-\bar{L}$ ) is at most countable, for every compact $K \subset L$. It follows by the 
Radon property of $\mu$ that $\bar{L}$ is $\bar{\mu}^{*}$-measurable with full $\bar{\mu}$-measure. On the other hand if $\bar{L}$ is $\bar{\mu}^{*}$-measurable and $\bar{\mu}(\bar{L})=\bar{\mu}\left(X^{*}\right)$ since $\bar{L} \cap X \subset L$, we deduce that $L \in \mathscr{F}$ and $\mu(L)=\mu(X)$. Hence the proof of the theorem is complete.

REMARKS 2.3. (i) Since every measure $\mu$ (with full support on a totally ordered space) has separable measure algebra, under the continuum hypothesis Theorem 2.1 follows also by Theorem 9 in [9].

(ii) Let $X$ be the space we obtain from $[0,1]$ by replacing each point $t \in(0,1)$ by two points say $(t, 0)$ and $(t, 1)$. We define an order " $<$ " on $X$ by

$$
(t, i)<\left(t^{\prime}, j\right) \quad \text { iff } t<t^{\prime} \text { or } t=t^{\prime} \text { and } i=0, j=1 .
$$

Clearly $(X,<)$ is a compact totally ordered space which supports nonatomic, Radon measures [[10, Example 4.4]. Clearly since $X$ has no two sided limit points, by Theorem 2.2, every non-zero, non-atomic Radon measure on $X$ provides an example of a totally ordered measure space which admits not almost strong liftings.

(iii) Theorem 2.2 is not valid for atomic measures. For example let $X$ be a compact totally ordered space which supports a non-atomic Radon measure $\nu$ such that the set $L$ of all two sided limit points is $\nu^{*}$-measurable with full measure and $X \neq L$. Then if $x \in X-L$ and $\mu=\nu+\delta_{x}$, we see that Theorem 2.2 is not applicable for $\mu$. (Here $\delta_{x}$ denotes the Dirac measure at $x$.)

(iv) In Theorem 2.2 the Radon property of $\mu$ cannot be omitted. To see this let $Y$ be the unit interval with the topology generated by all left-closed right-open intervals. It is known that $Y$ is a closed subspace of a totally ordered space $X$ (see Example 2.2(b) and Theorem 2.9 in [9]). Moreover, since the Borel sets of $Y$ and $(0,1)$ are the same, the Lebesgue measure $\lambda$ is a Borel measure on $Y$. Let $\mu$ be the extension of $\lambda$ on $X$. Then $\mu$ is a non-atomic measure on $X$ and $S_{\mu}=Y$. Further every point of $Y$ is a two sided limit point of $Y$, but $\mu$ admits not almost strong liftings (see Example 10 in [2]).

(v) The proofs of Theorems 2.1 and 2.2 apply unchanged for measures on generalized ordered spaces [9]

\section{REFERENCES}

1. A. G. A. G. Babiker, Lifting properties and uniform regularity of Lebesgue measure on topological spaces, preprint, University of Khartoum.

2. A. G. A. G. Babiker and W. Straub, Almost Strong Lifting and $\tau$-Additivity, Measure Theory, Proc. of the Conference held at Oberwolfach 1979, Lecture Notes in Math. 794 220-227, Springer-Verlag Berlin Heidelberg New York 1980.

3. H. R. Bennett and D. J. Lutzer, Separability, the countable chain condition and the Lindelöf property in linearly orderable spaces, Proc. Amer. Math. Soc., 23 (1969), 664-667. 
4. D. H. Fremlin, On two theorem of Mokobodri, note of June 1977.

5. L. Gillman and M. Henriksen, Concerning rings of continuous functions, Trans. Amer. Math. Soc., 77 (1954), 340-362.

6. S. Graf, On the existence of strong liftings in second countable topological spaces, Pacific J. Math., 58 (1975), 419-426.

7. A. Ionescu Tulcea and C. Ionescu Tulcea, Topics in the Theory of Lifting, Springer Verlag, Berlin-Heidelberg-New York 1969.

8. V. Losert, A measure space without the strong lifting property, Math. Ann., 239 (1979), 119-128.

9. D. J. Lutzer, On generalized ordered spaces, Dissertationes Math., 89 (1971), 5-36.

10. A. Sapounakis, Measures on totally ordered spaces, Mathematika, 27 (1980), 225-235.

Received October 19, 1981.

UNIVERSITY OF BRITISH COLUMBIA

VANCOUVER, B.C. CANADA V6T 1Y4 



\section{PACIFIC JOURNAL OF MATHEMATICS EDITORS}

DONALD BABBITT (Managing Editor)

University of California

Los Angeles, CA 90024

Hugo Rossi

University of Utah

Salt Lake City, UT 84112

C. C. Moore and Arthur Ogus

University of California

Berkeley, CA 94720
J. DugunduI

Department of Mathematics

University of Southern California

Los Angeles, CA 90089-1113

R. FinN and H. SAmelson

Stanford University

Stanford, CA 94305

\section{ASSOCIATE EDITORS}
R. ARENS
E. F. BECKENBACH
B. H. NeUmaNN
F. WolF
K. YoshidA (1906-1982)

\section{SUPPORTING INSTITUTIONS}

UNIVERSITY OF ARIZONA

UNIVERSITY OF BRITISH COLUMBIA

CALIFORNIA INSTITUTE OF TECHNOLOGY

UNIVERSITY OF CALIFORNIA

MONTANA STATE UNIVERSITY

UNIVERSITY OF NEVADA, RENO

NEW MEXICO STATE UNIVERSITY

OREGON STATE UNIVERSITY
UNIVERSITY OF OREGON

UNIVERSITY OF SOUTHERN CALIFORNIA

STANFORD UNIVERSITY

UNIVERSITY OF HAWAII

UNIVERSITY OF TOKYO

UNIVERSITY OF UTAH

WASHINGTON STATE UNIVERSITY

UNIVERSITY OF WASHINGTON 


\section{Pacific Journal of Mathematics}

\section{Vol. 106, No. $1 \quad$ November, 1983}

John Ballard, Clifford's theorem for algebraic groups and Lie algebras . . . . . 1

Philip Richard Bartick, II and Edwin Duda, Finite-to-one open mappings

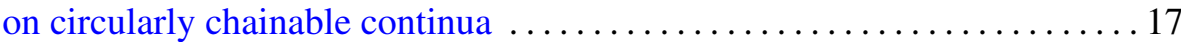

Frank Hayne Beatrous, Jr., $H^{\infty}$-interpolation from a subset of the

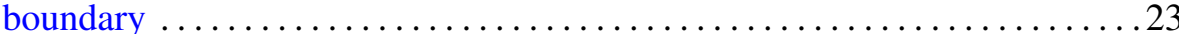

Sterling K. Berberian, Expectations in semifinite algebras ............ 33

Heron S. Collins and Wolfgang Ruess, Weak compactness in spaces of compact operators and of vector-valued functions $\ldots \ldots \ldots \ldots \ldots \ldots . \ldots 45$

David Downing and William O. Ray, Renorming and the theory of

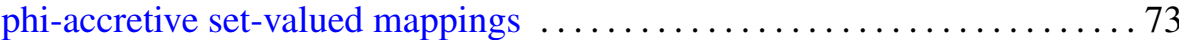

Harvey Charles Greenwald, On the theory of homogeneous Lipschitz

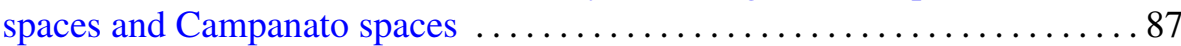

Irwen Valle Guadalupe and Lucio Ladislao Rodriguez, Normal curvature

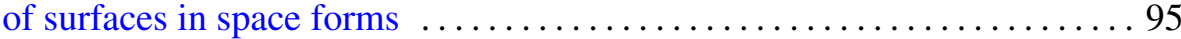

Boju Jiang, On the computations of the Nielsen number ............... 105

William H. Julian, Ray Mines, III and Fred Richman, Alexander

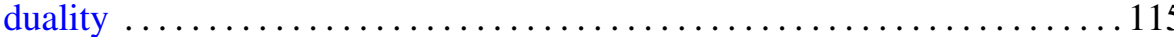

Midori Kobayashi, The connected component of the idèle class group of an

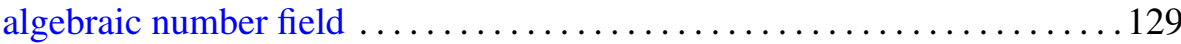

Ib Henning Madsen, Charles B. Thomas and C. Terence C. (Charles)

(Clegg) Wall, Topological spherical space form problem. III.

Dimensional bounds and smoothing

A. Sapounakis, The existence of strong liftings for totally ordered measure

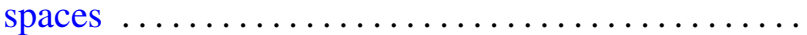

Gary Seitz, The root subgroups for maximal tori in finite groups of Lie type

Laurent Siebenmann and James M. Van Buskirk, Construction of irreducible homology 3-spheres with orientation reversing involution 